\title{
How many pixels make an image?
}

\author{
Antonio Torralba \\ Computer Science and Artificial Intelligence Laboratory, \\ Massachusetts Institute of Technology,
}

\begin{abstract}
The human visual system is remarkably tolerant to degradation in image resolution: human performance in scene categorization remains high no matter whether low resolution images or multi-mega pixel images are used. This observation raises the question of how many pixels are required to form a meaningful representation of an image and identify the objects it contains. In this paper, we show that very small thumbnail images at the spatial resolution of 32x32 color pixels provide enough information to identify the semantic category of real world scenes. Most strikingly, this low resolution permits observers to report, with $80 \%$ accuracy, $4-5$ of the objects that the scene contains, despite the fact that some of these objects are unrecognizable in isolation. The robustness of the information available at very low resolution for describing semantic content of natural images could be an important asset to explain the speed and efficiently at which the human brain comprehends the gist of visual scenes.
\end{abstract}

\section{Introduction}

In the images shown in Figure 1(a), we can easily categorize each picture into scene classes (a street, an office, etc.). We can also recognize and segment many of the objects in each image. Interestingly though, these pictures have only $32 \times 32$ pixels (the entire image is just a vector of 3072 dimensions with 8 bits per dimension), yet at this resolution, the images seem to already contain most of the relevant information needed to support reliable recognition of many objects, regions and scenes. This observation raises the question of how many pixels are needed to form a meaningful image. In other words, what is the minimal image resolution at which the human visual system can reliably extract the gist of a scene (the scene category and some of the objects that compose the scene)?

The gist of the scene (Friedman, 1979; Oliva, 2005, Wolfe, 1998) refers to a summary of a semantic description of the scene (i.e. its category, layout and a few objects that compose the scene). Such a summary may be extracted from very low-resolution image information (Oliva \& Schyns, 2000; Oliva \& Torralba, 2001) and, therefore, can be computed very efficiently. Low dimensional image representations, and short codes for 
describing images, can be important to explain how the brain recognizes scenes and objects very fast. VanRullen \& Thorpe (2001) have suggested that, given how fast recognition happens (150ms after stimulus onset), the first stages of recognition might be carried out by strictly feedforward mechanisms (see also Serre et al. 2007) in which neurons only have time to fire one or two spikes. They discuss that even with such a small amount of information, and when only a small fraction of the neurons fire one spike, it is possible to perform challenging recognition tasks (such as detecting the presence of animals in natural images). Bar (2007) suggests that low-spatial frequencies activate expectations that will facilitate bottom-up processing. In Torralba et al (2007), a low dimensional image representation is used to guide attention incorporating information about the scene context and task constraints.

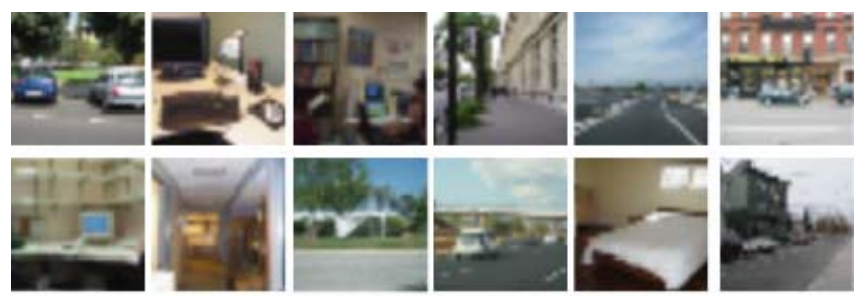

a) $32 \times 32$ scene thumbnails
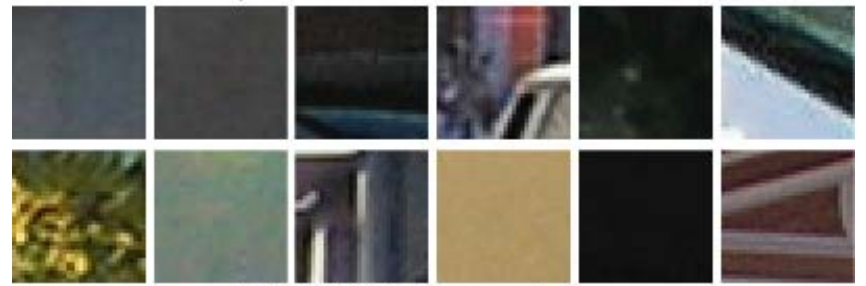

b) $32 \times 32$ image patches
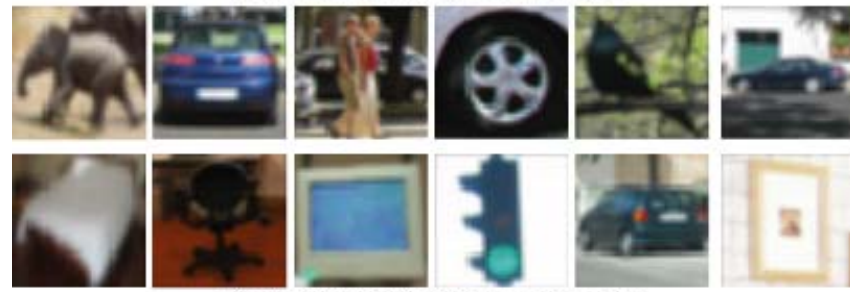

c) $32 \times 32$ object thumbnails

Figure 1: Scenes, patches and, objects all at 32x32 pixels. Note how rich the scenes (a) and objects (c) are in comparison with the image patches (b).

Studies on face perception (Bachmann, 1991; Harmon \& Julesz, 1973; Schyns \& Oliva, 1997; Sinha et al., 2006) have shown that when a picture of a face is down-sampled to a resolution as low as $16 \times 16$ pixels, observers are able to perform various face recognition tasks reliably (i.e. identity, gender, emotion). Remarkable performance with low resolution pictures is also found on scene recognition tasks (Oliva \& Schyns, 2000; Castelhano \& Henderson, 2008).

In this paper we study the minimal resolution required to perform scene recognition and object segmentation in natural images. Note that this problem is distinct from studies investigating scene recognition using very short presentation times and perception at a 
glance (Greene \& Oliva, in press; Joubert el al., 2005; Schyns \& Oliva, 1994; Oliva \& Schyns, 1997; Potter et al., 2002; Intraub, 1981; Rousselet et al., 2005; Thorpe et al., 1997; Fei-Fei et al., 2007; Rousselet et al., 2005; Renninger \& Malik, 2004). Here, we are interested in characterizing the amount of information available in an image as a function of the image resolution (there is no constraint on presentation time). In this work we will show that at very low resolutions, difficult tasks such as object segmentation can be performed reliably.

\section{Patches, objects and scenes}

Figure 1(b) shows 32x32 pixel patches randomly selected from natural images. A number of studies (Olshausen \& Field, 1996; Lee et al, 2003; Chandler \& Field, 2006) have focused on characterizing the space of natural images by studying the statistics of small image patches such as the ones shown in Fig. 2(b). Those studies helped to understand the receptive fields of visual neurons in early visual areas (Olshausen \& Field, 1996). However, many of these patches do not contain enough information to be recognized as part of a specific object or region as they contain flat surfaces or insufficient edges.

Figure 1(c) shows tight crops of objects rescaled at 32x32 pixels. These are the kind of images many times used in computer vision to train object detection algorithms. Olshausen et al. (1993) proposed an attentional system that selected 32x32 windows around regions of interest and argued that this was enough for recognizing most objects. Tight object and crops of objects, without background, have also been the focus of many studies in visual cognition. Many of those studies have focused on the study of faces, using image resolution as a way of controlling the amount of global and local information available.

Figure 1(a) depicts full scenes (what a human would typically see when standing on the ground and looking at a wide scene), scaled to 32x32 pixels. These scenes contain many objects which are surprisingly still recognizable despite the fact that they occupy just a few pixels each. The scene pictures used in this study have biases introduced by the way that photographers tend to take pictures. Although this could be considered as a drawback of our dataset, we think that the scene recognition and object segmentation tasks remain challenging and such biases are due to observer constraints and should be taken into account when coding images.

\section{Materials and methods}

The images used for this study were drawn from the scenes dataset from Oliva \& Torralba (2001) and the LabelMe database (Russell et al, 2008). In order to cover a large variety of different scenes, we collected 240 images evenly distributed within 12 scene categories. The scene categories included in this study are: 6 outdoor categories (street, highway, seaport, forest, beach and mountainous landscape) and 6 indoor categories (corridor, kitchen, bathroom, living-room, bedroom and office). All the images were originally of size $256 x 256$ pixels. 
For each image we generated low-resolution images at 4x4, 8x8, 16x16, 32x32, 64x64 and $128 \times 128$ pixels. In order to reduce the resolution of each image, we first applied a low pass binomial filter to each color channel (with kernel [ $\left[\begin{array}{lllll}1 & 4 & 6 & 4 & 1\end{array}\right]$ ) and then we downsampled the filtered image by a factor of 2 . Next, each pixel of the low-resolution images was quantized to 8 bits for each color channel. For visualization, the low resolution images were upsampled to 256x256 pixels.

Previous studies used a Gaussian filter in order to blur the images. The problem with using a Gaussian filter without downsampling the image is that it is difficult to evaluate the exact amount of information that is available to the observer. By first subsampling the image, the image resolution provides a clear upper bound on the amount of visual information available. In this paper we will use the size of the downsampled image as a measure of the amount of visual information that is available in the blurred images.

\section{Scene recognition}

Experiment

There were 28 naïve observers (age ranging from 18 to 40 years old) that took part in the scene recognition experiment. They all gave informed consent. The experiment had two conditions, color images and grayscale images: 14 observers participated in the color condition and 14 in the grayscale condition. Each image was shown at one of the 6 possible resolutions 4x4, 8x8, 16x16, 32x32, 64x64 and 128x128 pixels. All images were upsampled to 256x256 pixels for display and shown only once to each observer. The procedure consisted in a 12 alternative choice task: each image was categorized as belonging to one of the 12 possible scene categories. Participants where shown one example of each category in advance. The image was displayed on the screen until the participant made a choice by pressing one of the buttons associated to the 12 scene categories. Each participant saw a total of 240 images presented in random order.

\section{Results}

Figure 2 provides the overall pattern of results in the scene categorization task for the color and grayscale images as a function of image resolution. Below the graph, the top row of images illustrates the number of pixels at each resolution. The lower row shows the images that were presented to the participants during the experiment.

When images were shown at $128 \times 128$ pixels, performances were at ceiling, at $96 \%$ correct recognition rate. A few of the scene images in our dataset are ambiguous in terms of a unique scene category (like a road with a mountain which could be classified as a mountainous landscape or as a highway) therefore $100 \%$ recognition rate is impossible at this task. Chance level in this task is at $8.3 \%$. At a resolution of $4 \times 4$ pixels, performance for grayscale images was $9 \%$ and was not significantly different from chance $(\mathrm{t}(13)<1)$, but classification of color images was significantly higher than grayscale performance $(t(26)=3.8, p<0.001)$ with a correct rate of $18.4 \%$. 


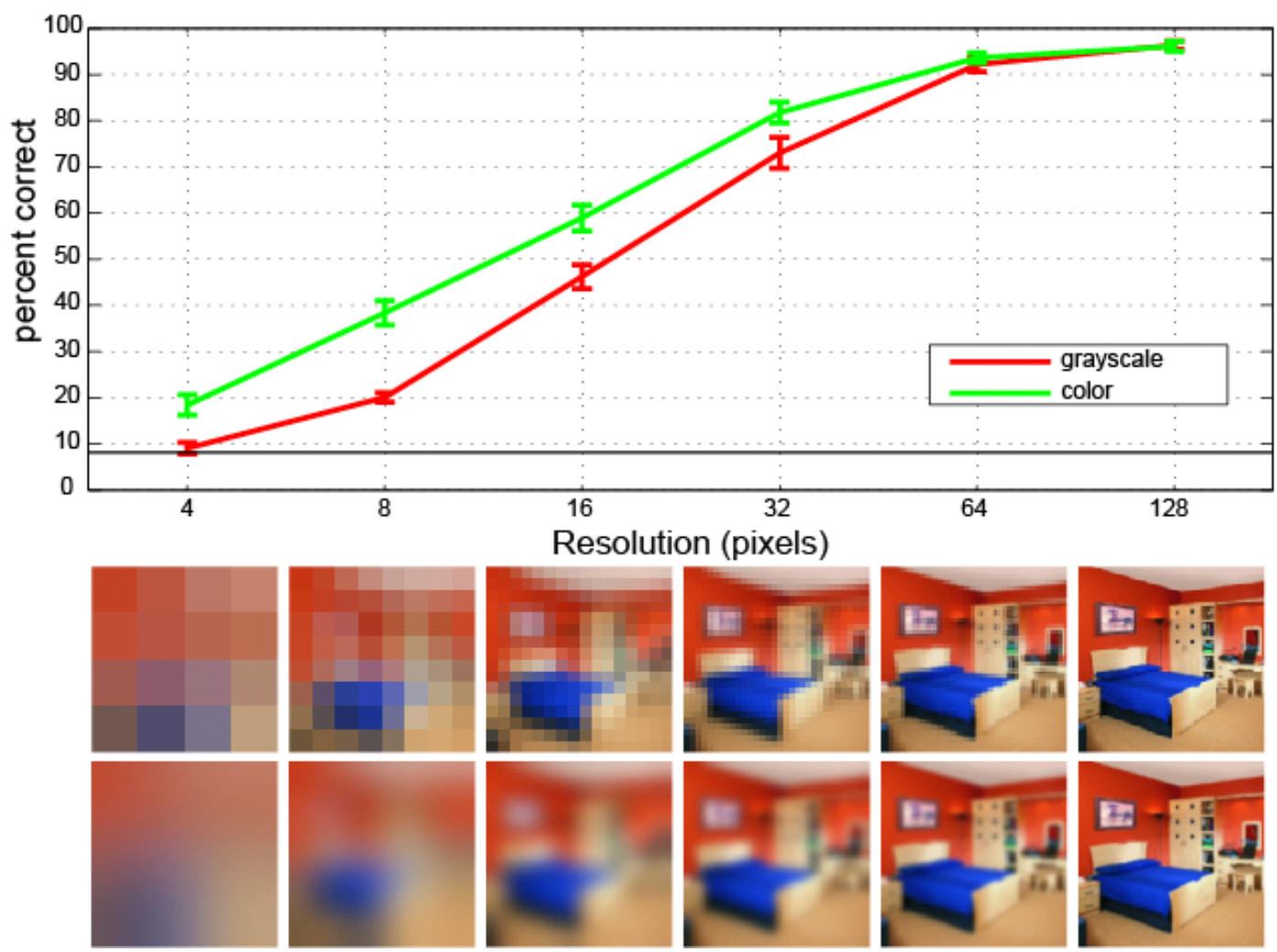

Figure 2: Scene recognition as a function of image resolution. Error bars represent one standard error of the mean, obtained from 12 participants for each condition. The vertical axis represents the correct recognition rate and the horizontal axis corresponds to the image resolution in a logarithmic scale. The black horizontal line represents chance level. The two rows of images illustrate the amount of information available at each resolution. The top row shows the downsampled images at each resolution (from $4 \times 4$, to $128 \times 128$ pixels) and the second row shows the images upsampled to $256 \times 256$ pixels that were shown to the participants.

At very low resolutions $4 \times 4$ and $8 x 8$, there was a strong bias towards selecting the beach category (20\% of the time) in both color and grayscale conditions. This response bias was present in early and late trials. At these low resolutions, images that belong to the categories highway and seaport were incorrectly classified as belonging to the beach category. For high resolutions there were no biases in the responses of the participants.

Scene recognition performance remains high even at relatively low spatial resolutions (in agreement with the results by Oliva \& Schyns, 2000). Participants had an $81.8 \%$ correct recognition rate when color images were presented at a resolution of 32x32 pixels. In out study, performance over $80 \%$ is found for an image resolution slightly higher than the one reported in Oliva \& Schyns (2000), who used a Gaussian filter and measured the frequency of $50 \%$ cut-off instead of the number of pixels in the downsampled image. For grayscale images, performance was at $73 \%$ at 32x32 pixels. As we lower the resolution, performances drop. There is a significant improvement in recognition accuracy when 
color is present (Oliva \& Schyns, 2000; Castelhano \& Henderson, 2008; Rousselet et al., 2005; Wurm et al 1993) for low resolution images.

The results presented in figure 2 were averaged over all scene types. However, it is important to note that different types of images will lose information at different rates as their resolution decreases. Figure 3 shows six images, at 6 resolutions, sorted according to the amount of image resolution needed to enable recognition. As the figure illustrates, some images can be recognized at extremely low resolutions (even when only 8x8 pixels are available), as the images on the left in Figure 3 , while others require higher resolution.
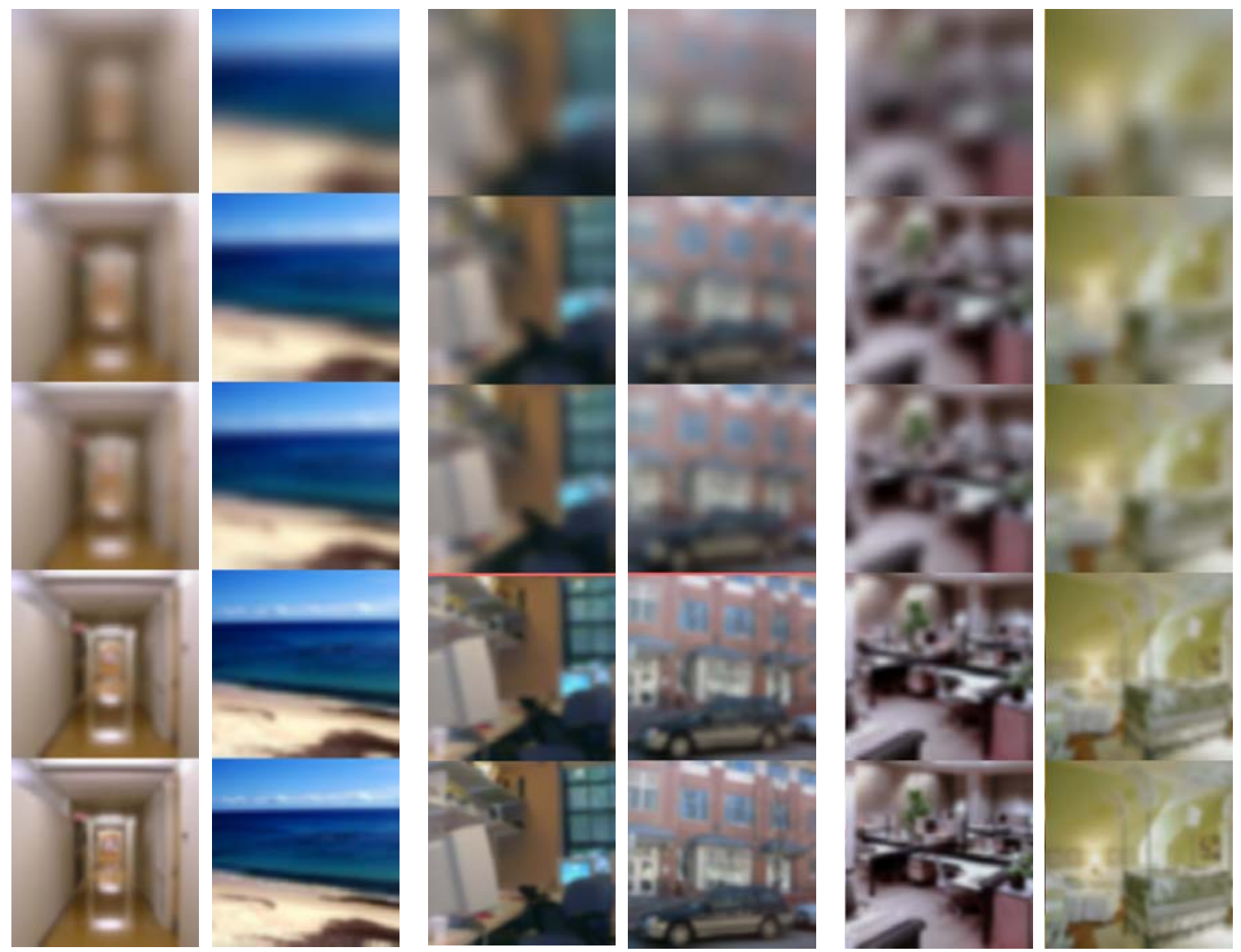

Figure 3: Images sorted by the amount of resolution required for becoming recognizable. Each row shows images that had been down-sampled to a resolution of $12 \times 12,18 \times 18,28 \times 28,42 \times 42$ and $64 \times 64$ pixels. The two images on the left (a corridor and a beach) are correctly categorized by most of the participants even at the lowest resolutions. The two images on the left require very high resolution in order to become recognizable (an office and a bedroom). The two images in the center need around 32x32 pixels in order to be recognized by most of the participants. Easy images are formed by few surfaces, and had diagnostic spatial layouts. 

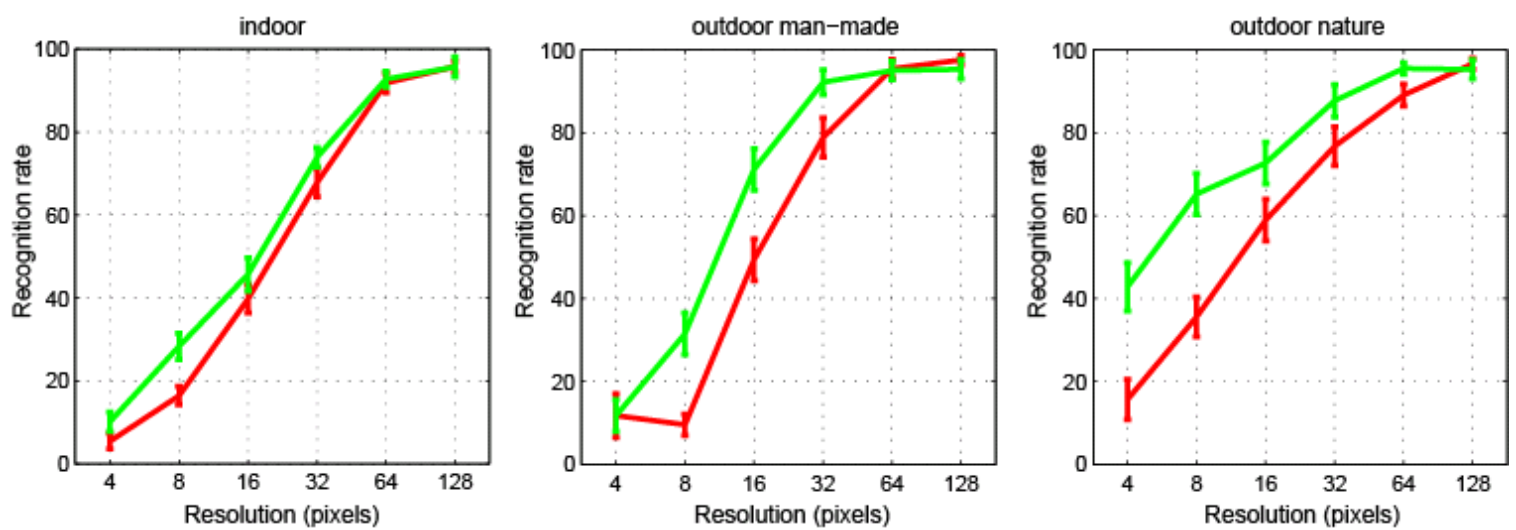

Figure 4: Performances for the 12-way classification task averaged over indoor scenes (6 categories), outdoor man made environments (street, sea port, and highway) and outdoor natural landscapes (beach, forest, mountainous landscape). Error bars correspond to the standard error.

Figure 4 shows the recognition performances averaged over three groups of scenes: indoor, outdoor man-made and outdoor natural scenes. Each of these super-ordinate scene groups has different requirements in terms of resolution and contribution of color information for the scene categorization task. Indoor scenes are the hardest classes of scenes to be recognized. First, we note that the contribution of color information for indoor scenes is marginal compared to the other scene types. Recognition rate at 32x32 pixels is at $77.8 \%$ correct for color images and at $67.8 \%$ for grayscale images. On the other hand, outdoor man-made scenes have a $92.2 \%$ correct recognition rate when presented in color at 32x32 pixels and $78.8 \%$ when presented in grayscale. For outdoor scenes, the inclusion of color information provides a great improvement on the recognition rate compared to the results on indoor scenes. A similar trend is observed for outdoor natural scenes, although for natural scenes recognition rate remains high even for very low resolutions (at $8 \times 8$ pixels, performance is still around 65.1\%).

Figure 5(a) provides the recognition performance as a function of image resolution for the 12 scene categories used in the experiment. Also, note that adding color information does not improve recognition performance with respect to grayscale images for most indoor categories. On the other hand, color provides a dramatic increase in recognition performance for natural environments, especially at very low resolutions. The contribution of color information is made clear in figure 5. Figure 5 shows the average of all the images that belong to each of the 12 scene categories. The top row represents the indoor categories. Only the corridor category can be easily recognized in this average image. The bottom row represents the 6 outdoor scene categories used in the experiments. Outdoor categories have a wider distribution of colors that are good predictors of the scene category. In a series of experiments, Oliva \& Schyns (2000) showed that diagnostic colors are important in many outdoor categories, particularly natural landscapes, as color of surfaces is a diagnostic feature of the scene category (see also Goffaux et al., 2005; Rousselet et al., 2005). However, color becomes less important to differentiate among man-made environments, where the color of objects and surfaces is often accidental and is not constrained by the function or material properties. 

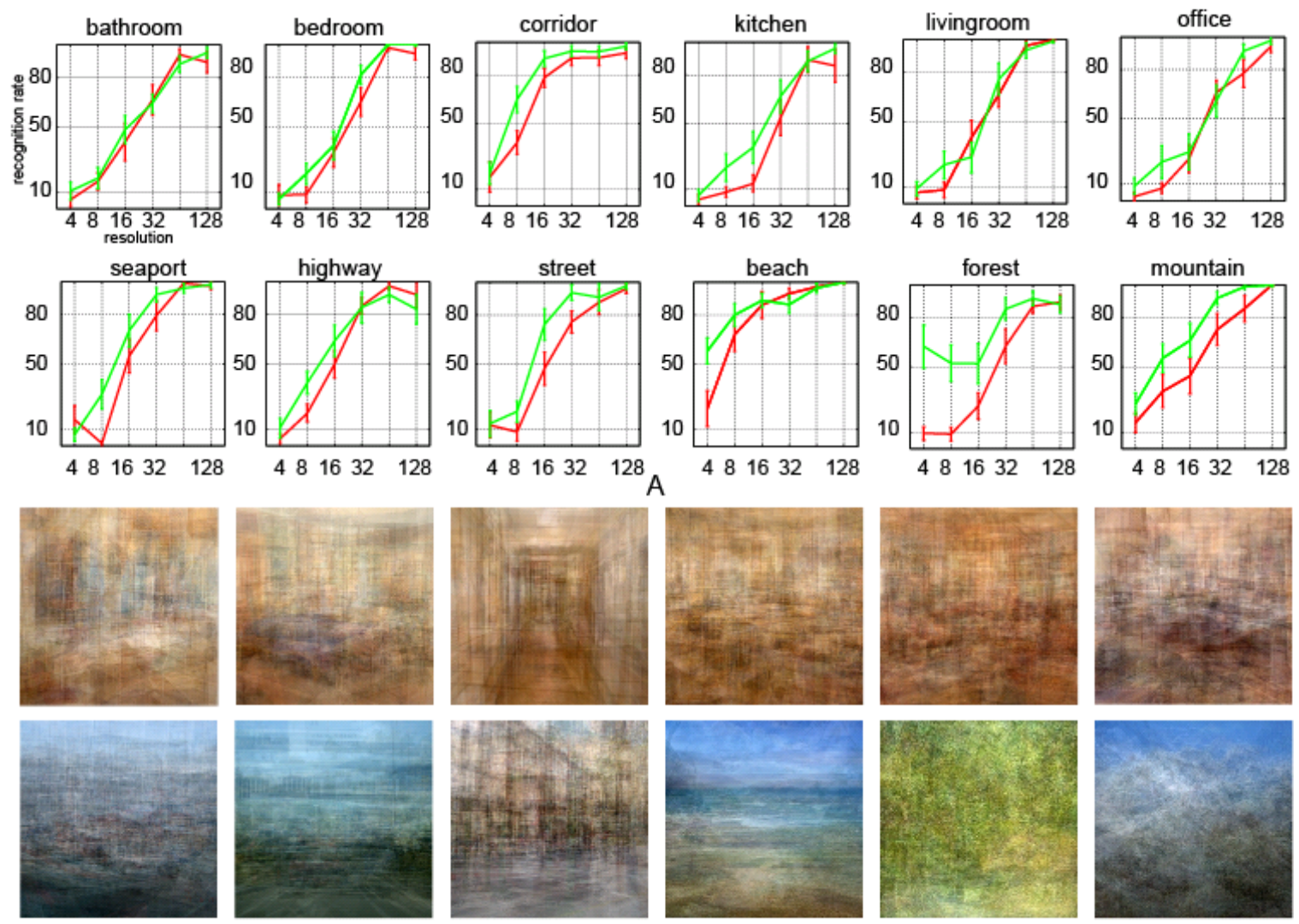

Figure 5: A) Performance on scene recognition for each scene category (chance is at $8.3 \%$ correct recognition rate) with grayscale and color images. Error bars correspond to the standard error. B) Average images of all the scene pictures that belong to each scene category. Each average is obtained and the pixel wise average of the 20 images in each group. The categories shown are (from left to right and top to bottom): bathroom, bedroom, corridor, kitchen, living room, office, seaport, highway, street, beach, forest and mountainous landscape.

An important observation is that not all the images can be correctly interpreted at very low resolutions. In order to study the distribution of resolutions needed to reach $80 \%$ recognition rate on single images, we performed an item analysis. As we do not have enough data to estimate the performance at each resolution independently, we use a psychometric function to estimate the relationship between recognition performance and image resolution. For each image we use logistic regression to fit the psychometric function (Klein 2001) relating probability of correct recognition as a function of image resolution (we use this function as it seems to fit the observed data, however, there are several other choices that are also valid). This function is defined by only two parameters $(a, b)$; therefore it can be estimated with only few samples.

$$
\mathrm{P}(\text { correct | resolution; a, b) = } 1 \text { / (1+exp(-a-b· log(resolution))) }
$$

The parameters of this psychometric function are estimated using maximum likelihood. Once the parameters are estimated we find the minimal resolution needed to reach $80 \%$ 
recognition rate for each image (this is the resolution for with $80 \%$ of the participants correctly recognized the scene category for a given picture).

Figure 6 shows the histogram of image resolutions needed to reach $80 \%$ recognition rate for the color and grayscale conditions. Only a very small percentage ( $3 \%$ with color) of the images need high resolution (more than 90x90 pixels). For color images, the maximum of the distribution is in the bin between $11 \times 11$ and 22x22 pixels. In that resolution interval, $28 \%$ of color images are recognized by $80 \%$ of the participants. For grayscale images $38 \%$ of the images are recognized when shown with a resolution between $22 \times 22$ and $45 \times 45$ pixels.

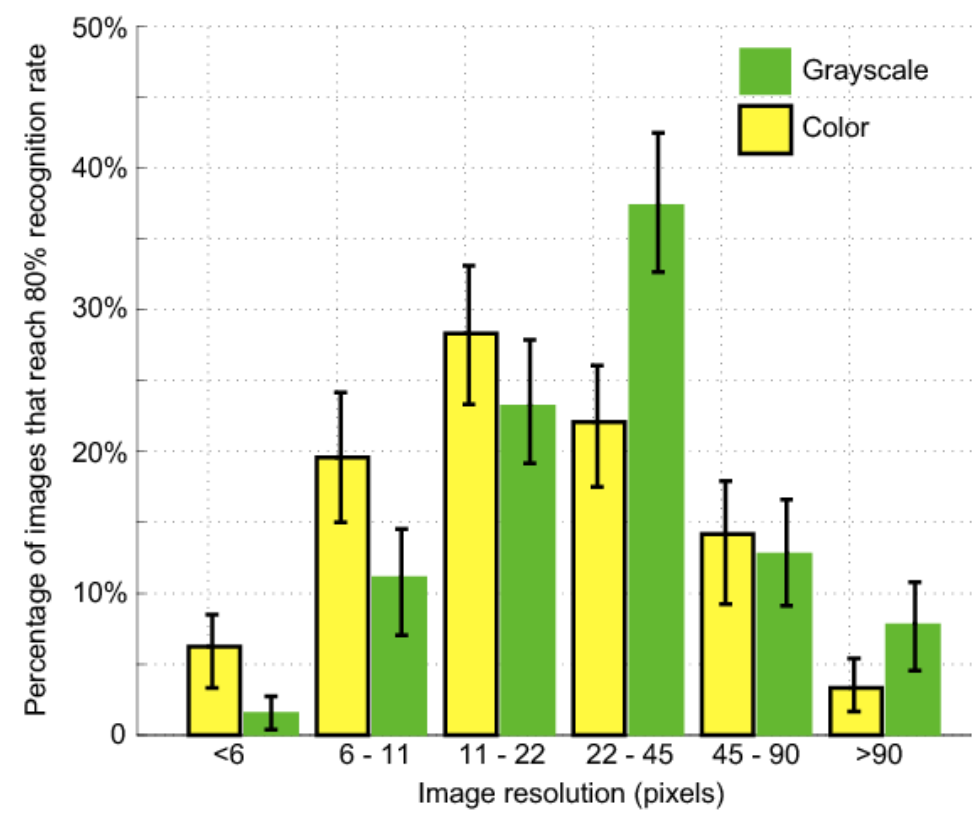

Figure 6: Distribution of image resolutions for which images are recognizable by $80 \%$ of the observers. For resolutions between $6 \times 6$ and $11 \times 11$ pixels, there are $20 \%$ of color images that are recognizable, while only $11 \%$ of the images can be recognized in that resolution range when shown in grayscale.

\section{Object segmentation}

\section{Experiment}

15 participants perform a total of 1195 trials. For each trial, an image was randomly selected from the set of 240 images described before. Each image was presented at one of the 6 possible resolutions $4 \times 4,8 \times 8,16 x 16,32 \times 32,64 \times 64$ and $128 \times 128$. For this experiment, images were presented in color.

Participants were first asked to provide the scene category of the image (this part of the experiment was identical to the scene recognition experiment described before), then, participants were asked to segment, using a drawing tool, as many objects and regions as 
they could recognize in each image. They traced and segmented one region/object at a time, entered its possible name, and then traced a second object, named it, etc. The 15 participants annotated a total of 5706 objects-regions.

In this experiment, participants were not given a list of objects to use. Therefore, once the experiment was concluded, we ran a validation procedure to decide which objects were correctly recognized. For this ground truth validation, each object was shown at the original resolution together with the annotation provided by one of the participants. For the validation, the information about the level of blur shown to the participant was not shown to avoid any bias. The images and participants were randomized for the validation stage. The validation was performed by the author.

\section{Results}

Figure 7(a) shows an example image and the segmentations produced by 6 participants at different resolutions. As the resolution of the image increases, the participants reported more objects and the reported objects had a higher probability of being correctly recognized. The question that we address here is to find what is the minimal image resolution needed so that participants can extract the information equivalent to the gist of the scene. Oliva (2005) and Wolfe (1998) argue that the gist of the scene might be composed of a coarse representation of the scene layout and a list of 4 or 5 objects.

Figure 7(b-c) summarizes the object recognition results for each of the three superordinate scene categories. Figure 7(b) gives the number of reported objects as a function of image resolution. As more resolution is available, more objects become recognizable. The number of objects reported seems to grow logarithmically with the image resolution for the three super-ordinate scene categories. Participants reported fewer objects for natural environments than for man-made environments. Figure 7(c) gives the recognition rate for the reported objects.

At a resolution of 32x32 pixels with color images, participants segmented and named 5 objects on average with $80 \%$ correct recognition rate (across all the super-ordinate scene categories). Figure 7(d) shows the distribution of sizes for the segmented objects. For all image resolutions most of the reported objects had an area that covered between 1/8 and $1 / 2$ of the image area. Figure 7(e) shows that the percentage of correctly recognized objects did not vary a lot between scales despite the large variation of object sizes. Figure 8(a) shows several examples of images at $32 \times 32$ pixels and the segmentations provided by the participants. Figure 8(b) shows some of the objects isolated from the scene. Some of the objects are defined by just a few blobs and recognition is only reliable when they are immersed in the scene. 

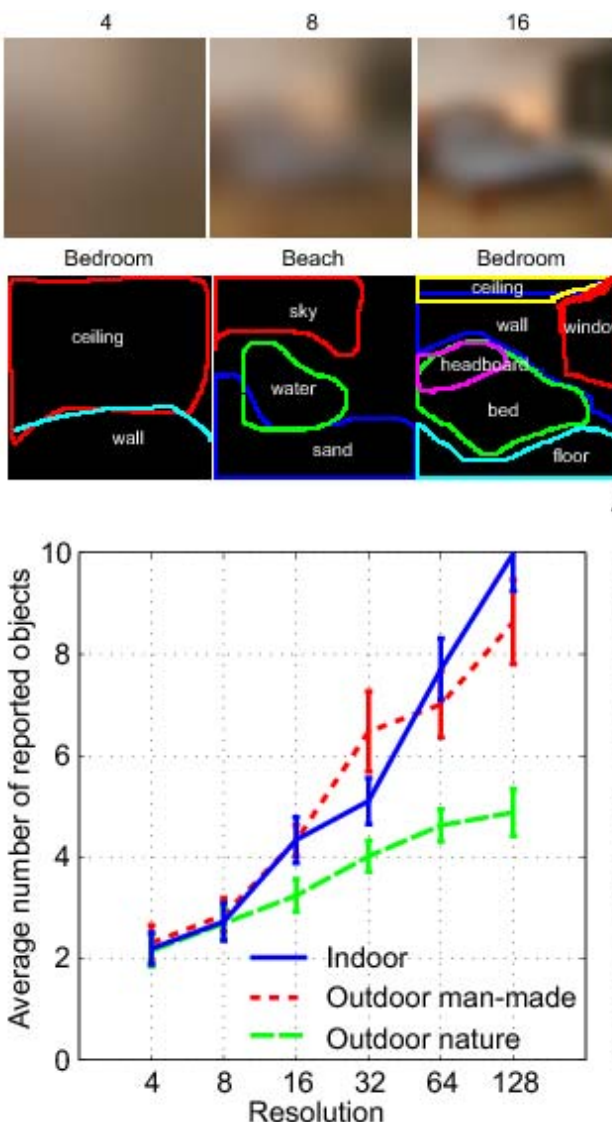

B

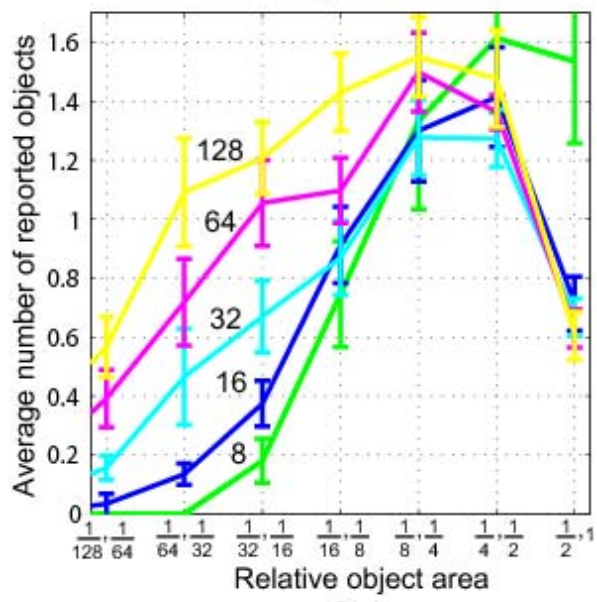

D

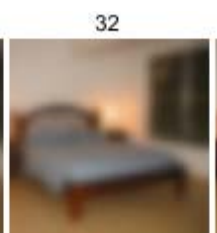

Bedroom

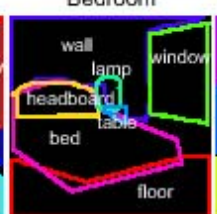

A

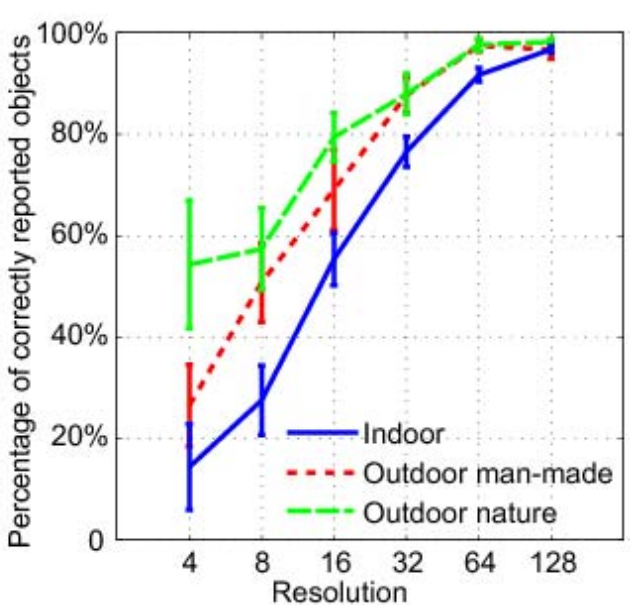

C

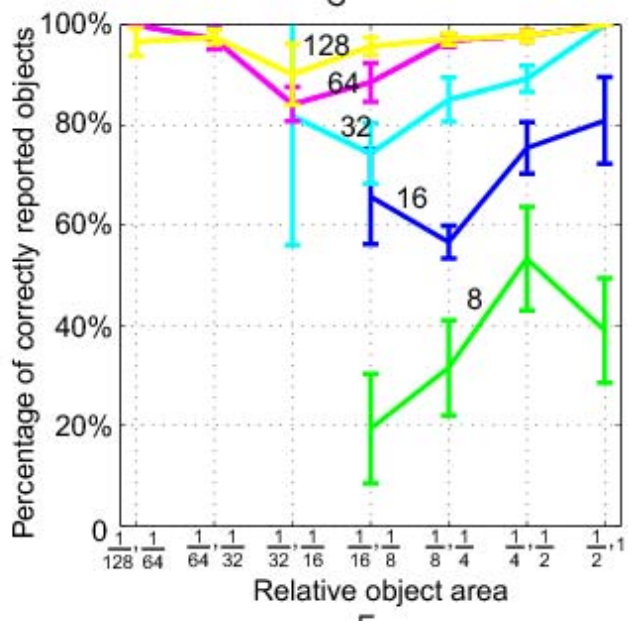

$\mathrm{E}$

Figure 7: As we increase the resolution, participants report an increasing number of objects. The number of objects reported per image seems to increase logarithmically with respect to the image resolution (B). From those objects, the object correctly recognized also increases with resolution reaching $80 \%$ around $32 \times 32$ resolution images (C). D) Average number of reported objects per image as a function of object size (measured as the proportion of the image occupied by the object). For all image resolutions, most of the reported objects cover an image area in the interval $[1 / 8,1 / 4]$ of the total image size. This is, between $12 \%$ and $25 \%$ of the image size. $E$ ) Recognition rate for reported objects as a function of their size on the image. 


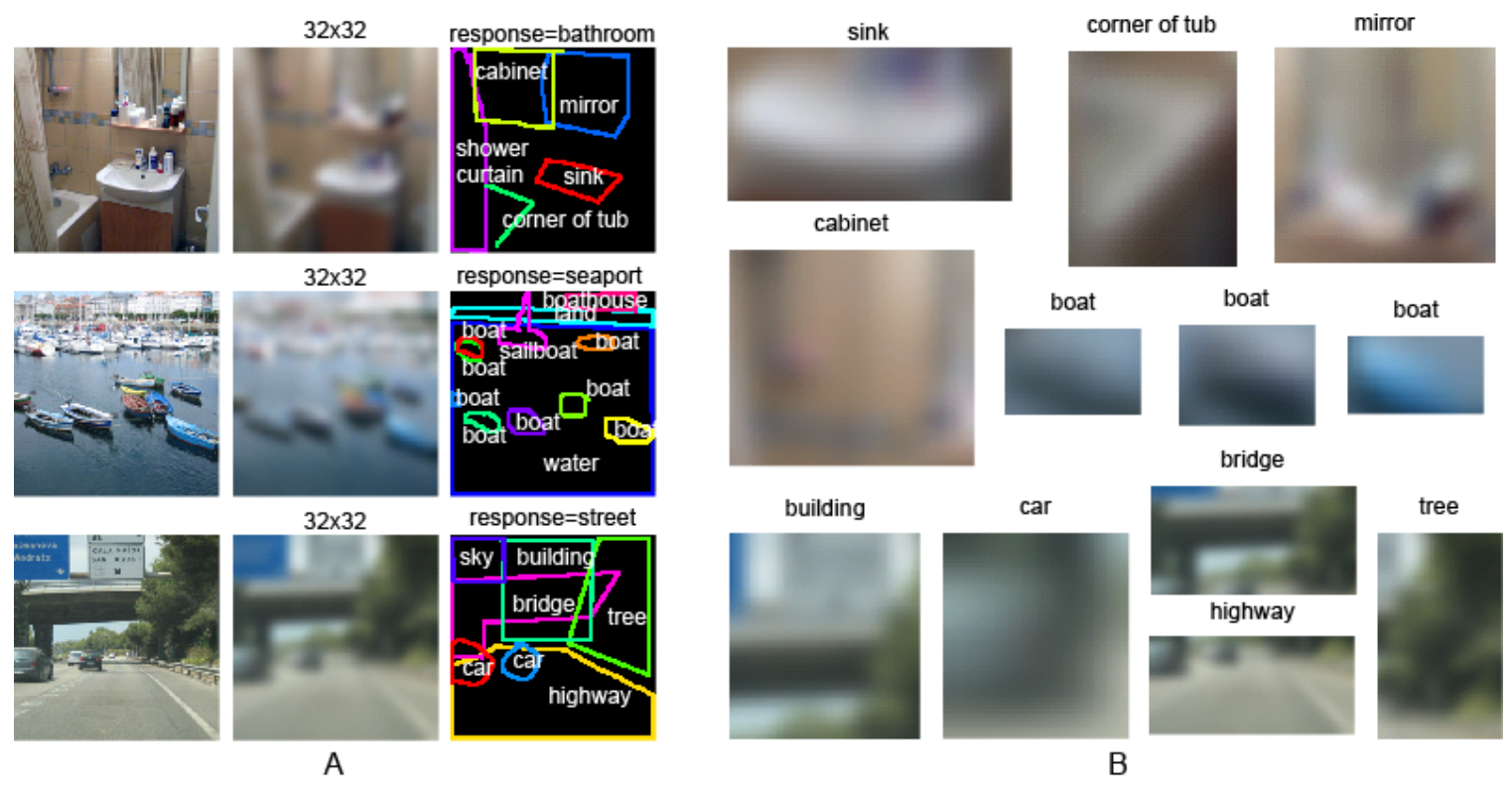

Figure 8: Images at a resolution of $32 \times 32$ pixels and the segmentations provided by the participants. Figure B shows some of the recognized objects cropped. Many of those objects become unrecognizable once they are extracted from the context.

At very low resolutions, recognition is heavily driven by contextual relationships between objects. This point is quantified in figure 9. Figure 9 shows that there is a significant interaction between performances on the scene recognition task and the object segmentation task. Figure 9 splits the object recognition results depending on whether participants identified correctly the scene prior to the object segmentation task. The number of objects reported did not change when participants miss-classified the scene category.

On average, for 32x32 images, participants segmented 5 objects independently on whether they assigned the correct or incorrect scene category to the picture. However, object recognition performance drops dramatically for low resolutions. At a resolution of 32 x32 pixels, the segmented objects were correct $89 \%$ of the times when the scene was correctly identified. Performances dropped to 53\% when the scene category was wrong. Failing to recognize the scene context has a major effect on object recognition performances at low image resolutions. At high-resolution, the effect is not so important. The same trends are observed when the performances are analyzed for the three superordinate scene groups (indoors, man-made outdoor and natural outdoor). 


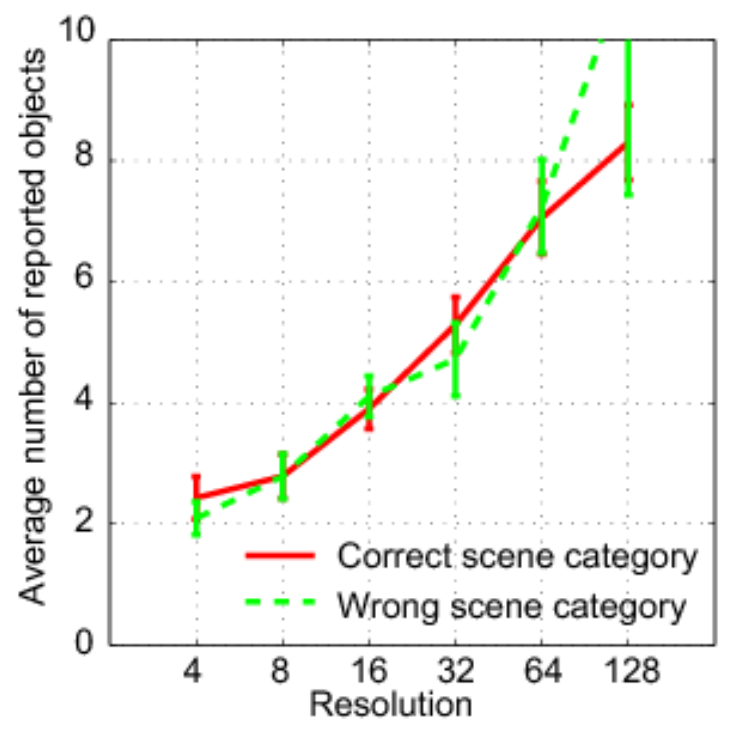

A

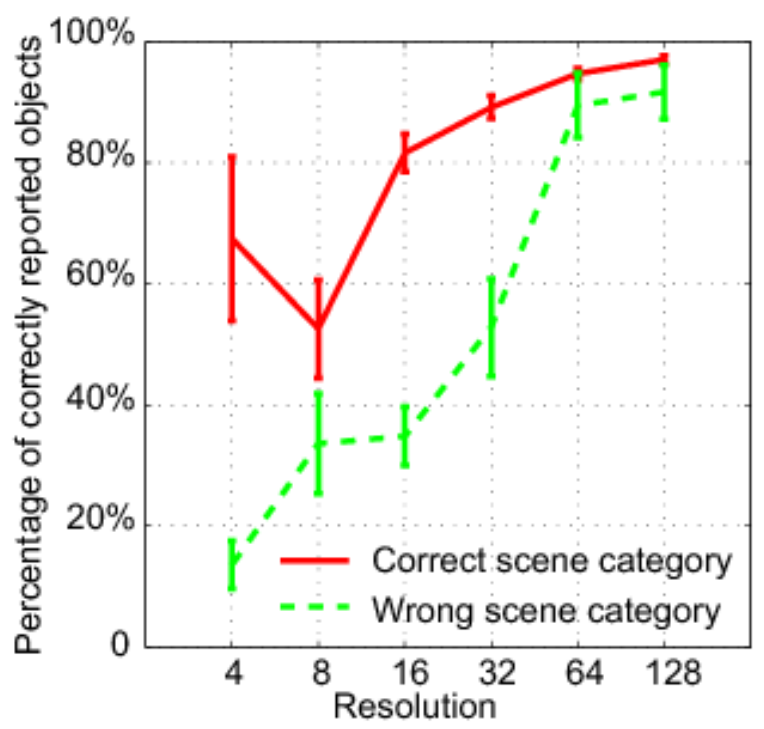

B

Figure 9: Performance on object recognition as a function of whether the scene was correctly identified or not. A) Number of objects reported as a function of image resolution, $B$ ) Recognition rate for reported objects as a function of resolution.

\section{Conclusion}

In this paper, we have explored two tasks, scene and object recognition as a function of image resolution. We have shown that $32 \times 32$ color images are already well formed pictures, with meaningful and recognizable global and local structures. Strikingly, for more than $80 \%$ of the images used in our experiments, the gist of the scene (the scene category and the identity and localization of 4-5 objects that compose the scene) is available with an image resolution of just 32x32 color pixels. Even, the lowest spatial frequency channels provide enough information for reliable recognition of the scene category, which can, in turn, be used to facilitate subsequent analysis of local regions and smaller objects in the scene.

How is recognition even possible at such low resolutions? A 32x32 pixel image represents $0.1 \%$ of the information available in a megapixel picture (similar to the number of nerve fibers in the optic nerve). Which image features can support recognition? At a resolution of 32x32 pixels most regular image features generally used to study the activation of the early cortical visual areas such as edges, junctions and textures are not available or are very weak. A lot of research effort has been devoted to understanding how early visual areas in the brain process fine image structures. However, very low resolution blobs, particularly colored blobs, provide an incredible amount of information that could guide the processing of high-resolution image detail (Schyns \& Oliva, 1994; Bar 2007). At low resolutions, the analysis of blob shapes and their spatial relationships become central for understanding the image content. A coding of the contextual relationships between regions is mandatory in order to achieve good recognition performance. 
From a computational view point, low-resolution images can be processed very fast and require small amount of memory (Torralba et al., 2008). The robustness of a short code for describing semantic content of natural images could be an important asset to explain the speed and efficiently at which the human brain comprehends the gist of visual scenes.

\section{Acknowledgments}

I would like to thank Aude Oliva, Rob Fergus and Bill Freeman for discussions and two anonymous reviewers for insightful comments. Funding for this research was provided by NSF Career award (IIS 0747120).

\section{References}

Bachmann, T. (1991). Identification of spatially quantized tachistoscopic images of faces: How many pixels does it take to carry identity? European Journal of Cognitive Psychology, 3, 85-103.

Bar, M. (2004). Visual objects in context. Nature Neuroscience Reviews. 5, 617-629.

Bar, M. (2007). The Proactive Brain: Using analogies and associations to generate predictions. Trends in Cognitive Sciences, 11(7), 280-289.

Castelhano, M.S., \& Henderson, J.M. (2008). The Influence of Color on Perception of Scene Gist. Journal of Experimental Psychology: Human Perception and Performance, 34, 660-675.

Chandler, D. M., \& Field, D. J. (2006). Estimates of the information content and dimensionality of natural scenes from proximity distributions. JOSA, 24:922-941.

Fei Fei, L., Iyer, A., Koch, C., \& Perona, P. (2007). What do we perceive in a glance of a real-world scene? Journal of Vision, 7(1), 1-29.

Friedman, A. (1979). Framing pictures: the role of knowledge in automatized encoding and memory of gist. Journal of Experimental Psychology: General, 108, 316-355.

Goffaux, V., Jacques, C., Mouraux, A., Oliva, A., Rossion, B., \& Schyns. P.G. (2005). Diagnostic colors contribute to early stages of scene categorization: behavioral and neurophysiological evidences. Visual Cognition, 12, 878-892.

Greene, M.R., \& Oliva, A. (in press). Recognition of natural scenes from global properties: seeing the forest without representing the trees. Cognitive Psychology.

Harmon, L. D., \& Julesz, B. (1973). Masking in visual recognition: Effects of twodimensional filtered noise. Science, 180, 1194-1197. 
Intraub, H. (1981). Rapid conceptual identification of sequentially presented pictures. Journal of Experimental Psychology: Human Perception and Performance, 7, 604-610.

Joubert, O., Rousselet, G., Fize, D., \& Fabre-Thorpe, M. (2007). Processing scene context: fast categorization and object interference. Vision Research, 47, 3286-3297.

Klein, S. A. (2001). Measuring, estimating, and understanding the psychometric function: A commentary. Perception \& Psychophysics. 63 (8), 1421-1455

Lee, A. B, Pedersen, K. S., \& Mumford, D. (2003).The nonlinear statistics of highcontrast patches in natural images. Int. J. Comput. Vision, 54(1-3):83-103.

Navon, D. (1977). Forest before the trees: the precedence of global features in visual perception. Cognitive Psychology, 9, 353-383.

Oliva, A., \& Schyns, P.G. (2000). Diagnostic colors mediate scene recognition. Cognitive Psychology, 41, 176-210.

Oliva, A., \& Torralba, A. (2001). Modeling the shape of the scene: a holistic representation of the spatial envelope. International Journal of Computer Vision, Vol. 42(3): 145-175.

Oliva, A. (2005). Gist of the scene. In the Encyclopedia of Neurobiology of Attention. L. Itti, G. Rees, and J.K. Tsotsos (Eds.), Elsevier, San Diego, CA (pages 251-256).

Oliva, A., \& Torralba, A. (2007). The role of context in object recognition. Trends in Cognitive Sciences, vol. 11(12), pp. 520-527.

Olshausen, B. A. et al., Anderson, C. H., and Van Essen, D. C. (1993) A neurobiological model of visual attention and invariant pattern recognition based on dynamic routing of information. J Neuroscience, 13, 4700-4719.

Olshausen, B.A., Field, D.J. (1996). Emergence of Simple-Cell Receptive Field Properties by Learning a Sparse Code for Natural Images. Nature, 381: 607-609.

Potter, M.C. (1975). Meaning in visual scenes. Science, 187, 965-966.

Rousselet, G. A. Joubert, O. R. Fabre-Thorpe, M. (2005). How long to get to the "gist" of real-world natural scenes? Visual Cognition, 12(6), 852-877.

Russell, B., Torralba, A., Murphy, K., \& Freeman, W. T. (2008). LabelMe: a database and web-based tool for image annotation. International Journal of Computer Vision, 77(3): 157-173. 
Serre, T., Oliva, A., \& Poggio, T. A. (2007). A feedforward architecture accounts for rapid categorization. Proceedings of the National Academy of Sciences, 104 (15), 64246429.

Sinha, P., Balas, B.J., Ostrovsky, Y., \& Russell, R. (2006). Face recognition by humans: 19 results all computer vision researchers should know about. Proceedings of the IEEE. Vol. 94, No. 11, 1948-1962.

Schyns, P.G. \& Oliva, A. (1994). From blobs to boundary edges: Evidence for time- and spatial-scale-dependent scene recognition. Psychological Science, 5, 195-200.

Thorpe, S., Fize, D., \& Marlot, C. (1996). Speed of processing in the human visual system. Nature, 381: 520-522.

Torralba, A., Oliva, A., Castelhano, M., \& Henderson, J. M., Contextual Guidance of Attention in Natural scenes: The role of Global features on object search. Psychological Review, 2006. 113(4): p. 766-786.

Torralba, A., Fergus, R., \& Freeman, W. T. (2008). 80 million tiny images: a large dataset for non-parametric object and scene recognition. IEEE Transactions on Pattern Analysis and Machine Intelligence.

VanRullen, R., \& Thorpe, S.J. (2001). Rate coding versus temporal order coding: what the retinal ganglion cells tell the visual cortex. Neural Computation, 13: 1255-83.

Van Rullen, R., \& Thorpe, S.J. (2001). The time course of visual processing: from early perception to decision making. Journal of Cognitive Neuroscience, 13(4), 454-461.

Walker Renninger, L., \& Malik, J. When is scene recognition just texture recognition? Vision Research, 44, 2004, pp. 2301-2311.

Wolfe, J.M. (1998) Visual Memory: What do you know about what you saw? Current Biology, 8: R303-R304.

Wurm L.H., Legge, G.E., Isenberg, L.M., \& Luebker, A. (1993). Color improves object recognition in normal and low vision. Journal of Experimental Psychology: Human Perception and Performance, 19, 899-911. 